\title{
Quantitative Determination of Material Hardness
}

\author{
Zdzislaw Pluta, Tadeusz Hryniewicz \\ Politechnika Koszalinska, Koszalin, Poland \\ E-mail:Tadeusz.Hryniewicz@tu.koszalin.pl \\ Received July 12, 2011; revised August 16, 2011; accepted August 22, 2011
}

\begin{abstract}
The paper contains description of a new quantitative method of evaluation of material hardness. First the essence of cognition subject, concerned with the hardness notion, has been discussed. Next some characteristics of exemplary existing evaluation methods have been presented. Then the attention was paid to the mistakes present in understanding/reasoning of the discussed problem. The revolutionary new method is given in the next part of the paper by presenting functional and parametric characteristics of the process of local deformation of a material. At the end, the justification - with the essential meaning for investigations of present and newer materials - to introduce one unified quantitative method of hardness evaluation, has been delivered.
\end{abstract}

Keywords: Hardness, Meyer's Law, Hardness Modulus, Young’s Modulus, Path Constant

\section{Introduction}

The problem of quantitative evaluation of solid (material) hardness is so vast/extensive that presentation of the whole existent knowledge would require several elaborations. At the same time, there are new methods proposed, by leaving apart some others, on how to determine and/ or improve them. It seems to be no need to analyze all the methods though some of them should be taken into consideration to present "the development" of the problem. First of all we should take apart the formation of newer and other methods leading to differentiation of the results.

There is lack of synthesis, with the new scientific actions going rather forward, without primary description of the natural reality. One should adequately quantitatively describe the phenomenon of local deformations of a material that is the essence of its hardness. Therefore let us return to the source! The development does not rely on uncritical acceptance of existent knowledge and continuous producing of new technical solutions. In the analysis, any erroneous well grounded principles or laws should be removed.

There is a law which does not reflect the reality in determination of material's hardness and that is the Meyer's law which will be presented here. Also some other rules, described using a function of the same class, which deform the reality should be removed.

A critical approach to the existent reality should pre- vail. In the paper, first the essence of hardness notion will be analyzed. Then the characteristics of exemplary methods of quantitative evaluation of material hardness will be given. After that, the erroneous reasoning will be presented. Afterwards the new method of determination of material hardness will be given. Functional and parametric characteristics of the process of local material deformation, being physical and measurable/determinable magnitude, will be presented.

Let us present the notion of hardness and indicate different understanding of this magnitude. For defining the hardness, the dictionary [1] includes definition of rigidity as "lack of rigidity, elasticity" and it is rather pseudodefinition. Furthermore, the same source [1] defines the hardness as "the magnitude of deformation occurred under action of concentrated forces (acting on a small area of the studied material)". There also, one of the measures of hardness is given, without an explanation of this notion. Some sources $[2,3]$ simply say that "the hardness of a material measures how tightly the atoms are held together within it" [2] or describe more extensively that "hardness is not an intrinsic property of any material, (like density or melting point), it is rather a characteristic deriving from the composition, the thermal and mechanical history of the material, and essentially from the structure (or more properly the microstructure) of the specimen involved" [3].

In [4] the following definition of hardness is given: "Hardness is the resistance of a material to a firm defor- 
mation resulting from indentation inside the intruder". There are several methods of hardness measurement, such as: Brinell, Rockwell, Vickers, Poldi, and Shore. It is worthy noting that hardness cannot be measured. Instead, it may be evaluated qualitatively as it is a sense magnitude. The measurement may be performed but only the measure of this magnitude with, for example, a unit thrust force (mechanical pressure) that is a force referred to the area of indentation arisen in a studied material. This method, determination of the physical magnitude (hardness measure), is called by Brinell.

The general definition of hardness may be précised as follows: Hardness of a material is its resistance to a local firm deformation. Then there are hardness measures (physical magnitudes), which should be measured (basic magnitudes), or determined (derived magnitudes, quite complex). There are different numerous methods concerning shape and hardness of an intruder, material dimensions, its structure, sample thickness, loading, manner of penetrator thrust on the studied material (static, dynamic, kinematic), to mention just a few of factors (classification identifiers).

There are several methods used for quantitative evaluation of material hardness. References [5-10] define several approaches to the problem, and here are some of them by: Brinell, Vickers, Rockwell, Chruszczow-Bierkowicz, Ludwik, UCI (Ultrasonic Contact Impedance), Shore, Poldi, Bauman, Leeba-RHT (Rebound Double Cone), Martens, Davydenkov, Ehrenberg, Bierbaum, Theinert, and Stöferle.

Amongst them there are the quantitative methods for hardness and micro-hardness evaluation: static, dynamic, scratch method. The static and scratch methods have been distracted and placed in the orbits (Figure 1) according to their authors' concepts.

There are the following static methods placed on the first orbit (Figure 1): HB-Brinell, HV-Vickers, HURockwell, HCH-Chruszczow-Bierkowicz, HL-Ludwik, $\mathrm{HG}-$ Grodziński. Second orbit is taken by scratch meth-

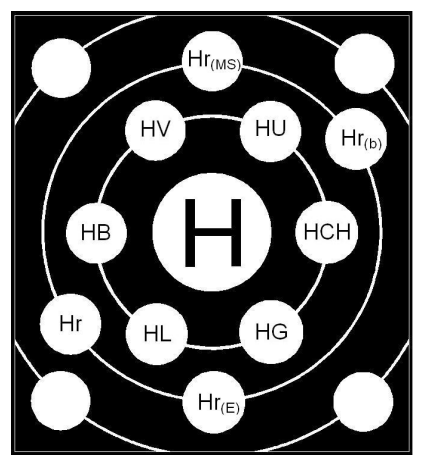

Figure 1. Orbits of the methods for hardness determination (notions in the text). ods such as: $H r_{(M S)}$-Martens, $H r$-Davydenkov, $H R_{(E)}$ Ehrenberg, $\mathrm{Hr}_{(b)}$-Bierbaum. Next orbits are shown to be taken by some potential methods, still waiting for their formation. It seems to be the effect of a scientist name to be included as well.

Some exemplary scratch methods rely on making a scratch by a set of standards of different hardness (file method, by Mohs), or by an indenter which is pressed perpendicularly to the surface and shifted at the same time.

The scratch hardness, according to Martens, uses a diamond cone of angle $90^{\circ}$. The loading $F$, to make a scratch $10 \mu \mathrm{m}$ wide, is the measure of hardness:

$$
H r_{(M S)}=F_{10}
$$

The scratch hardness, according to Davydenkov, uses a diamond cone of angle $90^{\circ}$. The inverse of scratch width $a \mathrm{~mm}$, obtained at loading $0.99 \mathrm{~N}$, is the measure of hardness:

$$
H r=\frac{1}{a_{50}}
$$

The scratch hardness, according to Ehrenberg, uses a diamond cone of any apex angle $\gamma$. The ratio of loading to the contact area of a material with the scratch during advance is the measure of hardness:

$$
H r_{(E)}=\frac{8 F \sin \frac{\gamma}{2}}{a^{2}}
$$

Any other man, say Mr Kowalski, could introduce his method relying on using a scratch of a regular ten-angle pyramid and the measure of hardness could be a square root of the scratch width loaded by $0.75 \mathrm{~N}$ force:

$$
H r_{(K)}=\sqrt{a_{75}}
$$

This way many other methods may be formed. Do we really need them?

The Meyer's law seems to be a sort of standard/indicator for the researchers to continue the study by using indenter intruding the studied material and to the quantitative evaluation of hardness. It is especially essential that though its inaccuracy is known [6,7], it is still in use. To say more, there are attempts to develop this law into the so called essential hardness $[9,10]$. According to this law, the force $F$ of ball intruding into material is a function of the obtained indentation with $d$ being the diameter of this indentation

$$
F=a \cdot d^{n}
$$

where $a$ and $n$ are proper constants obtained by experiment. 
The power form of the formula seems to be inadequate, and on the other hand, these two constants $a$, and $n$ have no physical interpretation. This formula is convenient and the logarithm may be found on both sides by obtaining the form of straight line in double logarithmic coordinates.

There are a few reasons of inexactness of the Meyer's formula [7] with the reasons being in other forms of formulae on hardness measures: they are e.g. formulae on HB (Brinell measure), HV (Vickers measure), HR'C $=\mathrm{HU}$ (measure corresponding with unified/standardized method [9]). One cannot use this Meyer's formula, supplement or extend it. Hardness should be described in a more accurate manner, and here this proper function will be an exponential function.

Let us present the hardness measure of a material as:

$$
H=a \cdot F^{n}
$$

By analyzing this measure of hardness one may notice that starting from some loading, its further increment does not affect the change in hardness. Let us another element is introduced to the Formula (6). This element of structure involves the essential hardness, and it is marked by symbol $H_{i}$. Therefore

$$
H=a \cdot F^{n}+H_{i}
$$

The details on this formula are given in the literature and sources cited therein.

\section{Adequate Law of Hardness}

Some exemplary works, proving of the quantum character of the reality, are given in [11-13]. Let us take into consideration a penetrator designed as a round straight cone of the apex angle $2 \alpha=120^{\circ}$. This penetrator advances vertically with the consecutive energetic states of deformed material (Figure 2). First, there is an initial stable state (ISS) and the tip of indenter is on the upper free surface of the object. This indenter continues to deform material reaching the position corresponding with an unstable state (US), the temporary/transient state where the thrust force $F_{o}$ of a tool attains a maximum value. During reverse motion of the penetrator, the reversal elastic deformation of the material occurs. This phenomenon ends on the depth corresponding with the final stable state (FSS) of the material.

In Figure 2 the lengths of material areas with characteristic phenomena are given. Symbol $H$ has been ascribed to the site where the material deformation (its loading as an active process) takes place. Section $H_{p}$ determines firm plastic deformation of a material.

All mentioned above states of material have been presented separately (Figure 3) to mark quantum nature of

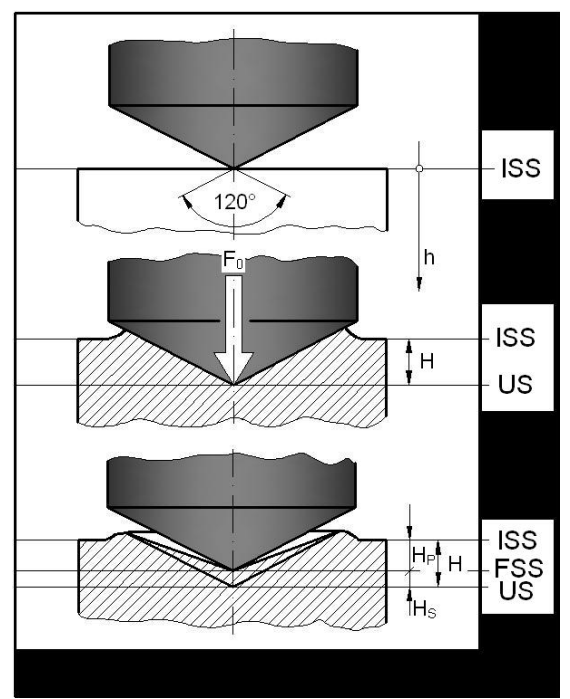

Figure 2. Positions of penetrator corresponding with particular energetic states of material.

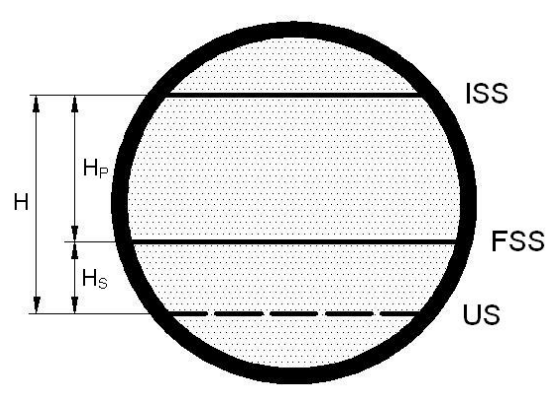

Figure 3. Sites of energetic states of a material during its deformation.

the natural reality.

There are lots of cases where exponential function of type $y=C x^{n}$ has been assumed as equivalent of a real course of a magnitude in spite if its inadequacy with reality. Here it is worthy mentioning the exponential Kassen formula on normal component of cutting force in machining with a single grain [14] which is still in use making much cognitive confusion. The Meyer's law also does not describe the reality that is the hardness of solids.

One should adequately describe the studied system. Let us consider the course of thrust force of a conic penetrator dependent on the coordination position of that device (Figure 4). The primary form of this plot has been excerpted from [15]. There are two branches: one of them, longer, corresponds to the system loading, whereas the second one, shorter and steeper, corresponds with deloading of the penetrator-material system. No power course may be observed. The analysis of the plot (Figure 4) indicates that only a determined exponential function should reflect it. 


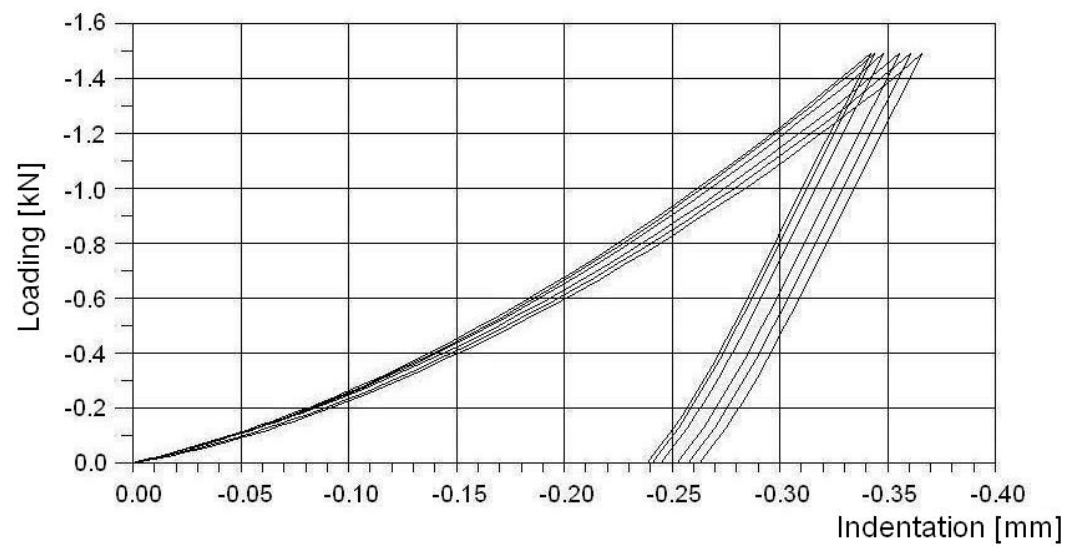

Figure 4. Registered course of thrust force of a conic penetrator on position coordinator of this tool [15].

Now let us return to the source, the differential description of the process. It has the following form under loading the system:

$$
\mathrm{d} F=\frac{\partial F}{\partial h} \mathrm{~d} h
$$

where symbol $F$ corresponds with loading force of the system, and $h$ is a coordinate of the position of penetrator tip.

One of the curves, central has been extracted (see Figure 4) and has been described (Figure 5) by equation being the solution of differential form as:

$$
F=F_{o}\left(\mathrm{e}^{\frac{h}{H_{o}}}-1\right)
$$

This equation concerns the phase of system loading that is the change of thrust force $F$ of penetrator on the length $H$ of its whole active path/way. Symbol $F_{o}$ denotes maximum thrust force and the mark $H_{o}-$ constant of active way.
Concerning the force $F_{o}$, its value should be assumed to correspond with the conditions of realized process. Constant $H_{o}$ is the magnitude of $H$ connected by a relationship resulting from substitution to the formula $F=F_{o}$ and obtaining

$$
H_{o}=\frac{H}{\ln 2}
$$

Now, a process parameter is to be determined that is one measure of hardness for all materials. That should allow for exact comparison of the studied materials under this feature which is called hardness. A conical diamond element of apex angle $2 \alpha=120^{\circ}$ is a penetrator.

The searched parameter results from differentiating the Equation (9). This operation leads to the solution as the next new physical magnitude, being the hardness modulus, i.e.

$$
M=\frac{\mathrm{d} F}{\mathrm{~d} h}=\frac{F_{o}}{H_{o}} \mathrm{e}^{\frac{h}{H_{o}}}=M_{o} \mathrm{e}^{\frac{h}{H_{o}}}
$$

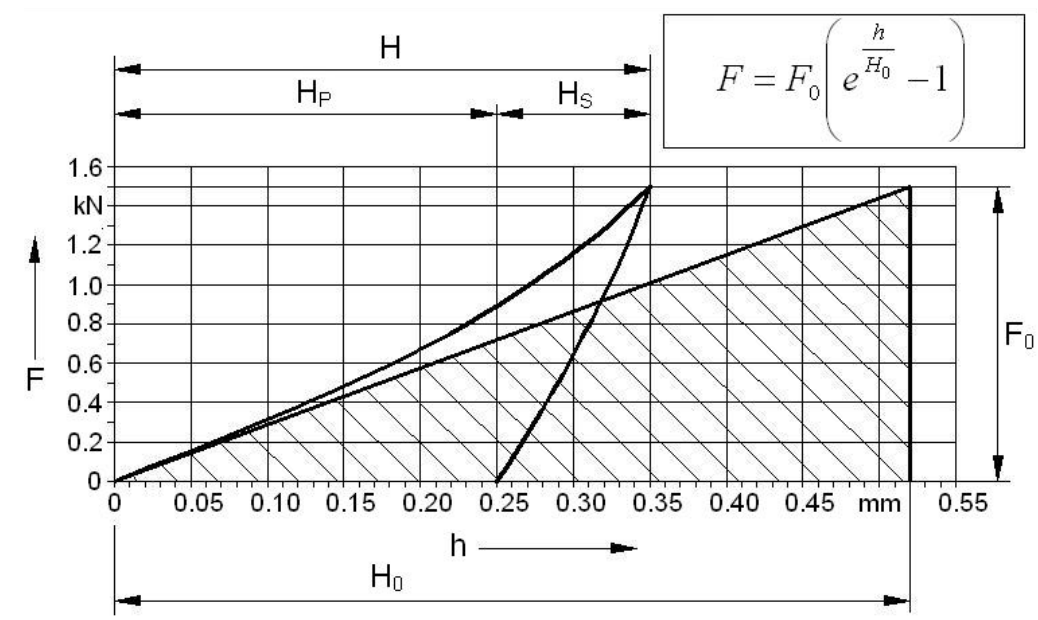

Figure 5. Characteristics of the process of local material deformation. 
where $M$ is this modulus, and $M_{o}$-energetic hardness modulus which, as results from the Formula (11), is determined as follows:

$$
M_{o}=\frac{F_{o}}{H_{o}}
$$

And after including (10)

$$
M_{o}=\frac{F_{o} \ln 2}{H}
$$

For cognitive purposes one may analyze the course of this new magnitude (Figure 6). This parameter could be assumed for practice.

Let us determine the energetic hardness modulus $M_{o}$ for a carbon steel ST3SY, with the plots of local deformation excerpted from [15] to use them for these analyses. Force $F_{o}$ was $1.5 \mathrm{kN}$, section length $H=0.35 \mathrm{~mm}$, so:

$$
M_{o}=\frac{1.5 \ln 2}{0.35}=\frac{1.5 \times 0.693}{0.35}=2.97 \mathrm{kN} \cdot \mathrm{mm}^{-1}
$$

The position of tangent to the curve $F=f(h)$ at its initial point at $h=0$, and the $M_{o}$ value may be determined straight from the Formula (12) or (13), if the curve is possibly regular as in the analyzed case. If the curve has an irregular shape/character, which is connected with the degree of uniformity of the studied material, then the obtained results should be worked out by statistics to deliver them to the tangent line.

This way a new adequate law of hardness is presented in Equation (9) with its specific form given in (11). They are hardness functional characteristics. The parametric characteristics are presented by Equation (12) or (13).

\section{Young's Modulus Determination}

Young's modulus of a studied solid may be determined based on the functional characteristics of the process of indentation of a panetrator in it with the characteristics referred to elastic materials deformations only. This may be presented by an analytical formula as follows:

$$
F=F_{o}\left[\exp \left(\frac{h-H_{p}}{H_{o}^{*}}-1\right)\right]
$$

where $H_{p}$ denotes the way length on which firm (plastic) deformations occur and $H_{o}^{*}$ is a constant of passive way determined by the relationship:

$$
H_{o}^{*}=\frac{H_{s}}{\ln 2}
$$

At this stage of considerations the joint analytical and graphical illustration of the whole process of local material deformation (Figure 7) may be presented. Figure 7 comprises also earlier described energetic states of the deformed material corresponding with the initial, intermediate (extremum) and final positions of the penetrator tip.

To determine Young's modulus, first the Hooke's law containing this modulus should be written:

$$
\sigma=\frac{F}{A}
$$

with

$$
\varepsilon=\frac{\Delta l}{l}
$$

where $F$ is the force the rod is deformed with, $A-\operatorname{rod}$ cross-section area, $\Delta l$-change of its length, and $l$ primary length of rod.

This discussion should be referred to the local deformation of material to find out the connection between parameters describing this process and Young's modulus. The force $F_{o}$ should be taken into account, and the area $A$ connected with the indentation cross-section at the height $H_{s}$. Therefore

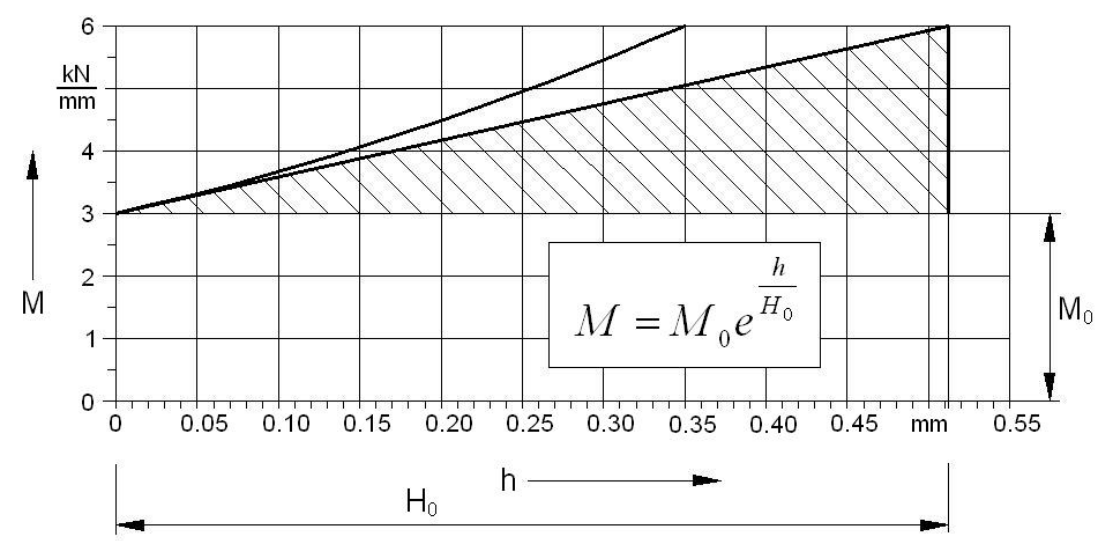

Figure 6. Course of the material hardness module. 


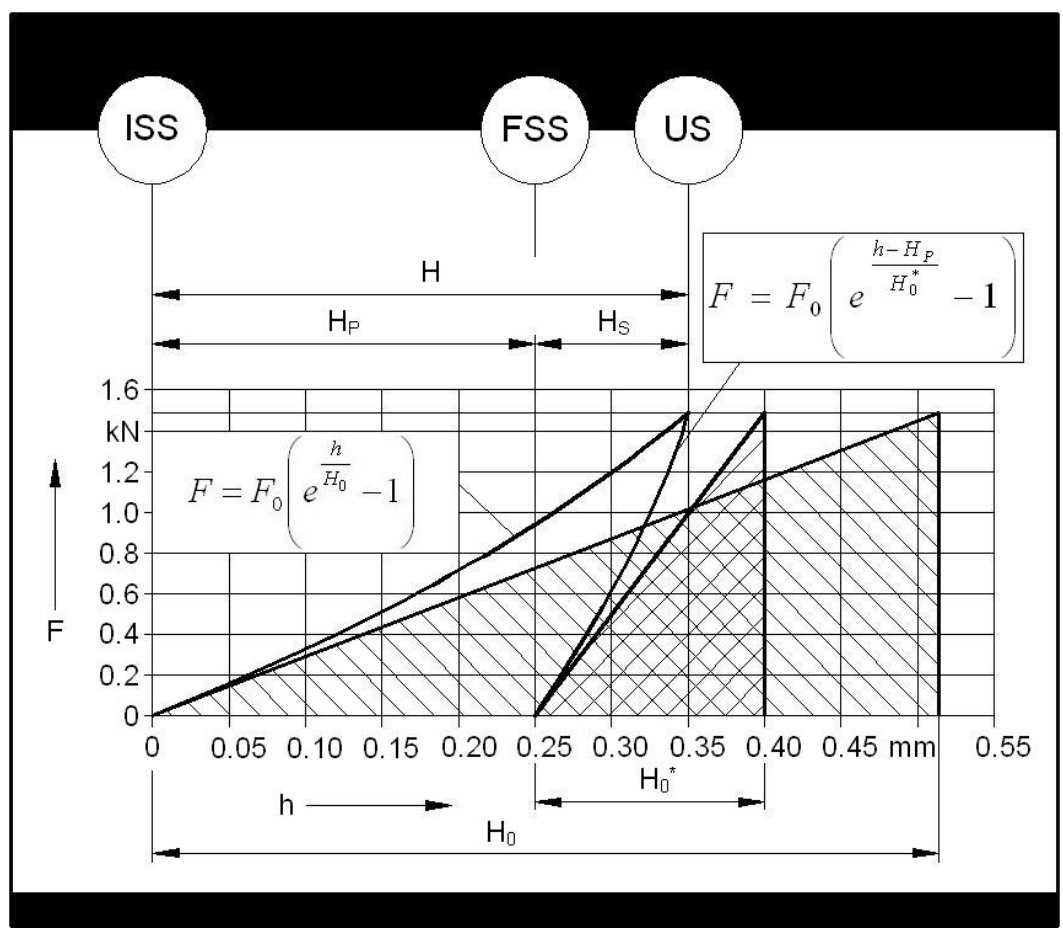

Figure 7. Graphical and analytical illustration of the whole process of local material deformation.

$$
A=\pi \cdot H_{s}^{2} \cdot \operatorname{tg}^{2} \alpha
$$

The relative deformation is described by the relationship

$$
\varepsilon=\frac{H_{s}}{H_{o}^{*}}
$$

And after taking into account (15)

$$
\varepsilon=\ln 2
$$

Thus after taking into account (19), the stress will be (see Formula 17) equal:

$$
\sigma=\frac{F_{o}}{\pi \cdot H_{s}^{2} \cdot \operatorname{tg}^{2} \alpha}
$$

And finely, after taking into account (21) and (22), the formula on modulus $E$ takes the form:

$$
E=\frac{\sigma}{\varepsilon}=\frac{F_{o}}{\pi \cdot H_{s}^{2}(\ln 2) \operatorname{tg}^{2} \alpha}=\frac{C F_{o}}{H_{s}^{2}}
$$

where

$$
C=\frac{1}{\pi(\ln 2) \operatorname{tg}^{2} \alpha}=1.38
$$

By presenting the literature data [13], concerning the considered process, we have: $F=1.5 \mathrm{kN} ; H=0.35 \mathrm{~mm}$; $H_{s}=0.1 \mathrm{~mm} ; 2 \alpha=120^{\circ}$. After calculation we obtain:

$$
E=206.8 \mathrm{kN} \cdot \mathrm{mm}^{-2}=2.07 \times 10^{5} \mathrm{MPa}
$$

\section{Procedure at the Determination of Energetic Hardness Modulus of Homogeneous Materials}

This procedure is quite simple and does not require any complex calculations. The mathematical statistics is limited to calculation of the mean values of determined magnitudes: the penetrator thrust force $F$ and respectively shifted (onto tangent direction to the process characteristics) coordinates $h^{*}$ of tool caving.

The problem is to be explained against freely assumed values of coordinates of characteristics points (Figure 8). They are quite dissipated that underlies non-homogeneity of material structure. Coordinates of force remain the same, and the coordinates of indentation $h$ are shifted onto tangent direction at initial point. These new positions, marked by a symbol $h^{*}$, result from the relationship of the following form:

$$
h^{*}=\frac{h}{\ln 2}
$$

The values of the magnitudes have been presented in Table 1. There is also given a formula on energetic hardness modulus of which form is adjusted to the introduced denotations. Then:

$$
M_{o}=\frac{\bar{F}}{\bar{h}^{*}}
$$

Its value was calculated and received $M_{o}=2.63$ 


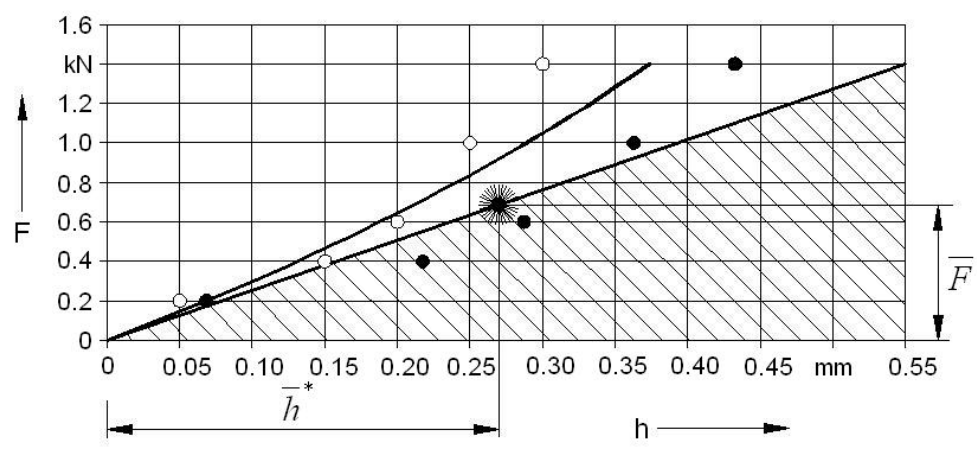

Figure 8. Illustration of the procedure at determination of energetic hardness modulus of inhomogeneous materials.

Table 1. Statement of the assumed and calculated magnitudes.

\begin{tabular}{cccc}
\hline$i$ & $F_{i}$ & $h_{i}$ & $h_{i}^{*}$ \\
\cline { 2 - 4 } & $\mathrm{kN}$ & 0.05 & 0.072 \\
2 & 0.2 & 0.15 & 0.216 \\
3 & 0.4 & 0.20 & 0.289 \\
4 & 0.6 & 0.25 & 0.361 \\
5 & 1.0 & 0.30 & 0.433 \\
$\Sigma$ & 1.4 & - & 1.371 \\
$\bar{F}$ & 3.6 & - & - \\
$\bar{h}^{*}$ & 0.72 & & 0.274 \\
& & $\bar{F}$ & 0.72 \\
& $M_{0}=\frac{\bar{h}^{*}}{0.274}=2.63 \frac{\mathrm{kN}}{\mathrm{mm}}$ & \\
\hline
\end{tabular}

$\mathrm{kN} \cdot \mathrm{mm}^{-1}$. It is worthy mentioning that having any $M_{o}$ value one may write functional characteristics of the process for any interstate space determined by the force $F_{o}$. It is enough now to determine the way constant $H_{o}$, and taking advantage of both magnitudes $\left(F_{o}, H_{o}\right)$, being a kind of supporting structure of all this type of functions, exponential functions, and present all their configurations.

\section{Summary}

One should underline that presented herewith the method of quantitative determination of material hardness is adequate in character and fully reflects the studied reality. It is quite simple and may successfully replace all other up-to-date existing methods which have no objective justifiable structure.

The delivered here energetic hardness modulus well characterizes resistance of a material on local firm deformations. It may replace all other measures of hardness used until today.

The only one energetic measure of hardness may al- low arranging/settling the problem and easing material characterization against its considered property. One method and one measure of hardness will allow proper evaluation of hardness characteristics of the studied materials. Material is the one variable magnitude.

Existing intensive progress in investigations of newer materials is an essential argument to introduce one unified quantitative method of hardness evaluation. This approach is quite general but should be concerned on all studied materials and objects.

\section{References}

[1] M. Szymczak, "Polish Dictionary (Słownik języka polskiego)," PWN, Warszawa, 1978.

[2] Engineered Materials. http://invsee.asu.edu/nmodules/engmod/prophardness.html

[3] E. E. Levi, "Practical Hardness Testing Made Simple," 2003. http://www.aws.org/educators/Library/0000/000587.pdf

[4] S. Okoniewski, "Metals Technology," PWSZ, Warszawa, 1960.

[5] L. Klimek, Ad. Rzepkowski and An. Rzepkowski, "Hardness in the Study of Machines," Laboratoria Aparatura Badania, Vol. 9, No. 1, 2004, pp. 12-17.

[6] S. Katarzyński, S. Kocańda and M. Zakrzewski, "Investigation of Mechanical Properties of Metals," WNT, Warszawa, 1967.

[7] F. Rudol, "Supplemented Meyer's Law-The Essence of Hardness of Solids," Mechanik, Vol. 48, No. 5-6, 1975, pp. 270-272.

[8] T. Pełczyński, "Relationship between E. Meyer's Power Coefficient and the Coefficient of Tensile Test," Mechanik, Vol. 40, No. 7, 1967, pp. 37-41.

[9] R. Rudol, "Standard Recommendation. Metals. Determination of Unification Hardness Using HR'C-HU Method," Mechanik, Vol. 35, No. 7, 1963, pp. 312-316.

[10] F. Rudol, "Interpretation of Hardness Study Results at Small Loadings. Essential Hardness of Solids," Zeszyty Naukowe Politechniki Poznańskiej, Mechanika, Vol. 30, No. 2, 1984, pp. 243-248. 
[11] Z. Pluta, "On the Oblique Flight of a Body across the Gravitation Space-Times," Nauka Innowacje Technika, Vol. 7-8, No. 5-6, 2004, pp. 58-61.

[12] Z. Pluta, "Of the d'Alembert's Rule with the Contents Permeated by Fiction and ... a Phantasy," Laboratoria Aparatura Badania, Vol. 9, No. 6, 2004, pp. 42-46.

[13] Z. Pluta, "On the R-F Scientific Chain," Koszalinskie Studia i Materiaty, Vol. 7, No. 1, 2004, pp. 131-143.
[14] G. Kassen, "Beschreibung der Elementaren Kinematik Les Schleifvorganges," Ph.D. Dissertation, RWTH Aachen University, Aachen, 1969.

[15] J. Bochnia, "Young's Modulus Determination Based on Statistic Test of the Cone Indentation," Archiwum Technologii Maszyn i Automatyzacji, Vol. 22, No. 2, 2002, pp. 41-48. 